\title{
Molekulare Allergiediagnostik - ein Muss für jeden fortschrittlichen Allergologen
}

— In kaum einem Gebiet der Medizin liegen Diagnostik und Therapie ähnlich nahe beieinander wie in der Allergologie, gelingt es doch in zahlreichen Fällen durch das eindeutige Auffinden der auslösenden Substanz und deren darauf folgender Meidung Beschwerdefreiheit zu erzielen. Deshalb legen die Lehrbücher der Allergologie üblicherweise großen Wert auf die Allergiediagnostik. Sie beruht auf vier Säulen:

- Anamnese,

_ Hauttestung,

_ In-vitro-Allergiediagnostik und

- Provokationstestung.

Wann immer durch einen der vorgenannten Schritte eine eindeutige Diagnose erzielt wurde, kann auf die Provokation verzichtet werden. Auch der Hauttest stellt im gewissen Sinne - eine Provokation, das heißt Belastung des Organs Haut dar und geht mit einem geringen Risiko systemischer anaphylaktischer Reaktionen einher. Andererseits gelingt durch die Anamnese allein die Diagnose einer Allergie nicht, selbst bei eindeutigem örtlichem und zeitlichem Zusammenhang. Es fehlt der Nachweis der immunologischen Sensibilisierung, die die Basis einer Allergie darstellt.

\section{Fortschritte in der molekularen Allergiediagnostik}

Die größten Fortschritte in der praktischen Allergologie haben sich in den letzten Jahren tatsächlich auf dem Gebiet der molekularen Allergiediagnostik ereignet. Darunter versteht man den Einsatz hoch gereinigter beziehungsweise rekombinant hergestellter Allergenmoleküle oder Teile derselben für die Diagnostik. Es ist deshalb außerordentlich zu begrüßen, dass PD Dr. Jörg Kleine-Tebbe und Prof. Dr. Thilo Jakob als Herausgeber den Band "Molekulare Allergiediagnostik" zusammengestellt haben, der dieses wichtige und komplexe Gebiet in der nötigen Tiefe umfasst und aus dem Blickwinkel des Klinikers hinsichtlich der Anwendbarkeit im klinischen Alltag darstellt.

Das Buch ist in drei Teile gegliedert: _ die Vorstellung der biologischen Familien und Allergenverwandtschaften,
_ die Beschreibung der unterschiedlichen Testsysteme im Labor und schließlich

_ die praktische Bedeutung molekularer Allergiediagnostik im allergologischen Alltag.

\section{Die wichtigsten Proteinfamilien der Allergene}

Im ersten Abschnitt werden die wichtigsten Proteinfamilien vorwiegend pflanzlicher Allergene beschrieben, nämlich die Verursacher der Baumpollenallergie und assoziierter Kreuzreaktionen anhand des Marker-Proteins Bet v 1 und Homologer. Profiline und Polcalcine werden als Beispiel von Pollen-Pan-Allergenen vorgestellt. Lipid-Transfer-Proteine und Speicherproteinen als stabile pflanzliche Nahrungsmittelallergene haben besondere Wichtigkeit für die klinische Risikobewertung. Ein weiteres Kapitel widmet sich den kreuzreaktiven Kohlenhydratepitopen, die lange Zeit wenig Beachtung fanden.

\section{Technologien und Prinzipien der Testsysteme}

Im zweiten Abschnitt werden die Testsysteme, ihre Technologie, die Prinzipien sowie die Unterschiede zwischen IgE-Einzelbestimmung (Singleplex) beziehungsweise Erfassung von IgE-Antikörpern gegen zahlreiche Allergene (Multiplex-Verfahren) vorgestellt und kritisch für den Einsatz im klinischen Alltag bewertet.

\section{Auslöser allergischer Erkrankungen}

Im dritten und umfangreichsten Abschnitt werden die Auslöser der wichtigsten allergischen Erkrankungen behandelt, so zum Beispiel die Marker- und Pan-Allergene bei Baum-, Gräser- und Kräuterpollenallergie. Am Beispiel der Erdnussallergie wird es am deutlichsten, dass die Molekular-Diagnostik echte Vorteile bringt. Ähnliches gilt für Allergien gegen Schalenfrüchte, bei Gemüse- und Fruchtallergie, bei nahrungsmittelabhängiger anstrengungsinduzierter Anaphylaxie oder bei der Insektengiftallergie. Weitere Kapitel umfassen den potenziellen Nutzen der molekularen Allergiediagnostik bei Allergien gegen Säugetiere, Fische, Hausstaubmilben, Latex
Jörg Kleine-Tebbe,
Thilo Jakob (HSG)

Molekulare Allergiediagnostik

Springer-Verlag Berlin Heidelberg 2015

Hardcover ISBN: 9783-662-45220-2

eBook ISBN 978-3-66245221-9

49,99 (Hardcover) bzw. 39,99 (eBook)

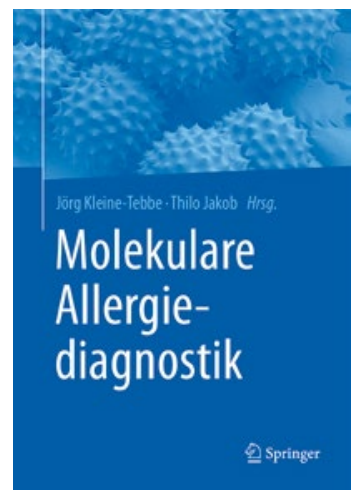

und Schimmel - Gebiete, auf denen es noch viel zu tun gibt.

\section{Didaktisch hervorragend}

In klar strukturierten und didaktisch hervorragend aufgearbeiteten Beiträgen besonders Lob gilt hier den umfangreichen und sehr anschaulichen Abbildungen - werden die wesentlichen Kenntnisse der modernen Allergenforschung für den klinisch tätigen Allergologen "übersetzt" und auf ihre Anwendbarkeit im klinischen Alltag überprüft. Die Autoren sind allesamt renommierte Experten aus dem deutschsprachigen Raum, die es verstehen, den molekular-allergologischen Hintergrund verständlich zu vermitteln und sowohl Nutzen als auch Grenzen dieser neuen diagnostischen Möglichkeiten aufzuzeigen.

\section{Von der Serie zum Buch}

Das Buch ist mehr als eine Aneinanderreihung von Einzelbeiträgen, auch wenn es ursprünglich so entstanden ist. Die zugrunde liegende Serie "Molekulare Allergologie" aus dem Allergo Journal war von Beginn an schon sehr sorgfältig und als ein großes Ganzes komponiert. Für das Buch haben die Autoren die meisten Beiträge noch erweitert und aktualisiert. Wer an moderner Allergologie interessiert ist und seinen Patienten diese zeitgemäße Diagnostik nicht vorenthalten will, sollte das Buch „Molekulare Allergiediagnostik" sein Eigen nennen. Es ist ein Muss für jeden fortschrittlichen Allergologen!

Prof. Dr. Dr. Johannes Ring 